\title{
Diabetic patients treated with dialysis: complications and quality of life
}

\author{
V. R. Sørensen - E. R. Mathiesen - T. Watt • \\ J. B. Bjorner • M. V. N. Andersen • B. Feldt-Rasmussen
}

Received: 19 June 2007 / Accepted: 11 July 2007 / Published online: 18 September 2007

(C) Springer-Verlag 2007

\begin{abstract}
Aims/hypothesis The aim of this study was to describe the prevalence of complications, health-related quality of life (HRQOL) and the influence of beliefs about control over health in diabetic dialysis patients.

Methods Of 53 eligible diabetic patients on chronic dialysis during January 2004 in our clinic, 38 (76\%) completed a kidney-specific (Kidney Disease Quality of Life) and a generic (SF-36) questionnaire and were characterised in terms of cardiovascular diseases and diabetic complications. Matched groups of non-diabetic dialysis patients $(n=$ 40) and diabetic patients with a long duration of diabetes and normal kidney function $(n=38)$ served as controls. Generic HRQOL was compared with matched data from a survey on the Danish general population $(n=2248)$.
\end{abstract}

Electronic supplementary material The online version of this article (doi:10.1007/s00125-007-0810-1) contains supplementary material, which is available to authorised users.

V. R. Sørensen $(\bowtie) \cdot$ B. Feldt-Rasmussen

Department of Nephrology, Copenhagen University Hospital,

Blegdamsvej 9,

2100 Copenhagen, Denmark

e-mail: vr.soerensen@rh.dk

E. R. Mathiesen - T. Watt

Department of Endocrinology, Copenhagen University Hospital,

Copenhagen, Denmark

\section{J. B. Bjorner}

National Research Centre for the Working Environment,

Copenhagen, Denmark

M. V. N. Andersen

Department of Ophthalmology, Copenhagen University Hospital, Copenhagen, Denmark
Results Micro- and macrovascular complications were significantly more frequent in diabetic dialysis patients than in diabetic patients without renal disease. Self-rated physical health was significantly worse $(p<0.01)$ in diabetic dialysis patients $(35 \pm 9$ [mean $\pm \mathrm{SD}])$ compared with nondiabetic dialysis patients $(41 \pm 10)$, diabetic patients with normal kidney function $(45 \pm 12)$ and the matched general population $(47 \pm 19)$. The diabetic dialysis patients had similar levels of kidney-specific quality of life and mental health compared with the control groups. Reduced physical health was predicted by the presence of end-stage renal disease, diabetes and short time spent in education. Among the diabetic patients, those who believed more on their own ability to control their diabetes and less on chance reported better mental health and were less likely to be on dialysis. Conclusions/interpretations Diabetic dialysis patients are characterised by a high prevalence of diabetic complications, reduced self-rated physical health but relatively good mental health.

Keywords Diabetes - Diabetic complications - Dialysis . End-stage renal disease $\cdot \mathrm{KDQOL} \cdot$ Locus of Control · Maculopathy $\cdot$ Quality of life $\cdot$ Retinopathy $\cdot$ SF-36

$\begin{array}{ll}\text { Abbreviations } \\ \text { CABG } & \begin{array}{l}\text { coronary artery bypass grafting } \\ \text { chronic kidney disease }\end{array} \\ \text { CKD } & \begin{array}{l}\text { end-stage renal disease } \\ \text { ESRD }\end{array} \\ \text { HRQOL } & \begin{array}{l}\text { health-related quality of life } \\ \text { KDCS }\end{array} \\ \text { kidney disease component summary } \\ \text { KDQOL } & \text { Kidney Disease Quality of Life } \\ \text { MHLC-C } & \begin{array}{l}\text { Multidimensional Health Locus of Control, } \\ \text { FCS }\end{array} \\ \text { Form C } \\ \text { mental component summary }\end{array}$


PCS physical component summary

PCI percutaneous coronary intervention

SF-36 Medical Outcomes Study 36-Item Short-Form Health Survey

\section{Introduction}

The number of diabetic patients referred for renal replacement therapy in Denmark has been stable since 2003 [1]. This may be due to the implementation of a multi-factorial and more intensive reno-protective intervention in patients with diabetes. The impact of this intervention among diabetic patients on chronic dialysis is still subject to debate [2]. In a recent study of 23,000 diabetic haemodialysis patients it has now convincingly been shown that poor glycaemic control is associated with a significantly increased mortality rate [3].

Although the survival of diabetic patients treated with dialysis in Denmark has improved during the past 15 years [4], a significant number of diabetic patients treated with chronic maintenance dialysis still suffer from one or more diabetic complications besides the kidney failure and have increased cardiovascular morbidity and mortality rates $[5,6]$.

Because of a shortage of donor kidneys for renal transplantation, and the fact that only a minor proportion of patients with diabetes, particularly those with type 2, who have end-stage renal disease (ESRD) are eligible for transplantation, most diabetic patients with ESRD will be on maintenance dialysis for the rest of their life. Healthrelated quality of life (HRQOL) is therefore an important parameter in this group of patients. Furthermore, the identification of factors that contribute to poor quality of life is important, when quality of life of dialysis patients with diabetes is to be improved.

The mechanisms that cause some diabetic patients to progress to ESRD requiring dialysis and allow others to have normal urinary albumin excretion for more than 10 years are not fully understood. Large clinical trials have demonstrated that several therapeutic strategies in the treatment of diabetic patients are effective in slowing or even preventing the progression to ESRD, as well as other diabetic complications [7-12]. However, if such complex treatment regimens and demanding self-care activities are to be effective, a high degree of patient adherence and compliance must be ensured. Several studies have documented that this is not easily achieved [13-15]. A study on the implementation of guidelines for the prevention of diabetic nephropathy in type 1 diabetic patients found that $79 \%$ did not achieve the target BP of $\leq 140 / \leq 90 \mathrm{mmHg}$ [16]. Good glycaemic control, defined as $\mathrm{HbA}_{1 \mathrm{c}}<7.5 \%$, was only seen in $24 \%$ of the patients [16]. There may be many reasons for these problems with compliance. A known important predictor of good compliance is the patient's own confidence in their ability to control their disease [17-19], which in psychology has been described by the term 'locus of control'. Locus of control defines the attitudes of people with regard to the control they have over their life. Research investigating locus of control has traditionally classified individuals into two categories: internal (own influence) and external (chance, powerful others and doctors influence) believers. Individuals with a high internal score believe that one's own behaviour determines one's health status. External control has been conceptualised as the belief that outside forces such as chance/luck, powerful others in one's network, or healthcare providers are responsible for health status and outcome. A combination of a high internal score and a low external score has been associated with better metabolic control and better adherence to diabetes self-care regimens [20-23].

The general aim of the present study was to identify potentially relevant intervention areas in the treatment and care of diabetic dialysis patients. Specific aims were to (1) describe the prevalence of diabetic and cardiovascular complications; (2) compare the quality of life of diabetic dialysis patients with that of non-diabetic dialysis patients, diabetic patients with normal kidney function and the general population; and (3) describe locus of control in the two groups of diabetic patients.

\section{Methods}

Patients

In this case-control study, diabetic patients, who were treated with chronic dialysis therapy during January 2004 at Department of Nephrology, Copenhagen University Hospital were selected as the cases. There were two control groups: a group of non-diabetic dialysis patients, matched to the cases in terms of age, dialysis modality and duration of ESRD (nondiabetic dialysis controls), and a group of diabetic patients with a diabetes duration of $>10$ years and an urinary albumin excretion rate of $<30 \mathrm{~g} / \mathrm{l}$, matched to the cases in terms of age and type of diabetes (diabetic controls).

Diabetic patients treated with dialysis There were 65 diabetic patients on chronic dialysis therapy (56 with haemodialysis, nine with peritoneal dialysis). Nine patients did not understand Danish sufficiently to participate in the HRQOL part of the study, and three patients suffered from dementia. The remaining 53 patients were asked to complete the Kidney Disease Quality of Life Short Form, (KDQOL-SF) version 1.3 questionnaire and the Multidi- 
mensional Health Locus of Control questionnaire, Form C (MHLC-C) (copies of the questionnaires in both Danish and English can be found in the Electronic supplementary material), and $38(72 \%)$ patients answered the questionnaires sufficiently and were included in the study.

Non-diabetic dialysis controls Forty (75\%) out of 53 matched non-diabetic dialysis patients answered the KDQOL-SF 1.3 questionnaire and were included in the study as non-diabetic dialysis controls. In all dialysis patients the questionnaires and information about the study were given during a haemodialysis session or at an ordinary visit at the peritoneal dialysis outpatient clinic by either the first author or by an assistant.

Diabetic controls The generic component of the KDQOLSF questionnaire (Medical Outcomes Study 36-Item ShortForm Health Survey [SF-36]) and the MHLC-C questionnaire were further administered as mail-out/mail-back questionnaires to 76 diabetic outpatients who fulfilled the inclusion criteria and were matched to the cases with respect to age but were not treated with dialysis. A reminder was sent to nonresponders after 4 weeks. A total of $38(50 \%)$ patients completed the questionnaire and were included in the study as diabetic controls. The ethics committees of Copenhagen and Frederiksberg approved the study and all patients gave informed consent.

General population SF-36 data regarding the general population were obtained from the National Health and Morbidity Survey 2005, which was carried out by the Danish National Institute of Public Health and comprised a total of 22,486 individuals [24]. For this study, we used the respondents living in the same area as the patients $(n=2248)$.

\section{Clinical data}

The demographic and clinical data included age, sex, dialysis modality, duration of diabetes, type of diabetes, duration of ESRD, primary cause of ESRD, presence of current foot ulcers, previous amputations, history of acute myocardial infarction or coronary artery bypass grafting (CABG) or percutaneous coronary intervention (PCI), history of stroke, presence of angina pectoris, BP, BMI and dialysis access. Demographic, clinical and biochemical data were obtained from the patients' records. Haemoglobin, cholesterol and serum albumin were routinely measured in all patients every month, $\mathrm{HbA}_{1 \mathrm{c}}$ every third month, and $\mathrm{Kt} / \mathrm{V}$ (a method of measuring dialysis adequacy, where $\mathrm{K}=$ clearance, $\mathrm{t}=$ dialysis time and $\mathrm{V}=$ volume of distribution) every sixth month. For diabetic patients a copy of the latest eye examination was obtained and the presence of retinopathy and vision of the best eye with correction were recorded. Patients with a best corrected visual acuity of $\leq 0.3$ (impaired vision) were recorded. Presence of maculopathy was recorded and retinopathy was classified as none, background (mild non-proliferative, moderate nonproliferative or pre-proliferative changes) or proliferative by an ophthalmologist. All clinical data were also obtained from the patients' records for all patients who did not respond to the questionnaires.

\section{Quality of life}

HRQOL was measured using the Danish version of the questionnaire KDQOL-SF, which consists of a kidney disease-specific part (KDQOL) [25] and a generic part (SF-36). The KDQOL-SF has been used in several studies of dialysis patients [26-29]. The English version has previously been translated into Danish and validated in Danish dialysis patients [30-33]. Dialysis patients completed both questionnaires, whereas diabetic patients with normal kidney function only completed the SF-36. The kidney-specific part measures 11 multi-item scales (symptoms, effect of kidney disease, burden of kidney disease, cognitive function, work status, sexual function, quality of social interaction, sleep, social support, dialysis staff encouragement and patient satisfaction), and scales range from $0-100$, with a higher score indicating better health for all scales. A kidney disease component summary (KDCS) was calculated as the mean of the 11 multi-item scores. If one or more scores were missing, the average of the remaining scores was calculated. The KDQOL data were scored according to the manual for the KDQOL (available from http://www.gim.med.ucla.edu/kdqol/, last accessed in August 2007). The eight SF-36 multi-item scales measure different dimensions of health (physical function, role limitations caused by physical problems, bodily pain, general health, vitality, social function, role limitations caused by emotional problems, and mental health) and the scales range from $0-100$. A higher score indicates better health for all scales. In addition, these eight dimensions were combined into two summary scores: The physical component summary (PCS), which measures physical health, and the mental component summary (MCS), which measures mental health. The PCS and MCS are standardised to the American general population, with a general population mean of 50 and an SD of 10 [34]. The SF-36 data were scored according to the procedure of the Danish manual for SF-36 [35]. We also included questions regarding smoking habits and education (short, 7 years in municipal primary school; medium, 9 or 10 years in municipal primary school; long, grammar school). 


\section{Locus of control}

Diabetic patients also completed the MHLC-C, which is a condition-specific locus-of-control scale that can be adapted for use in any medical condition (in this study, diabetes). This questionnaire provides an assessment of beliefs about control over health, and has been used in studies concerning compliance and adherence $[21,25,36]$. The instrument comprises 18 items rated on a six point Likerttype scale ranging from 'strongly disagree' to 'strongly agree', with four subscale scores: 'internal', 'chance', 'doctors' and 'powerful others'. Higher scores indicate greater belief in that subscale domain in relation to health (diabetes). The scores range from 6-36 in the 'internal' and 'chance' scales, and from 3-18 in the 'doctors' and 'powerful others' scales.
Statistical analyses

Continuous variables were compared using the Student's $t$ test or the Mann-Whitney $U$ test, depending on the distribution, and categorical variables were compared using $\chi^{2}$ analyses. The SF36 scores were reported as means $\pm \mathrm{SD}$, and were analysed using the Student's $t$ test and multiple regression methods. To evaluate the effect of assuming that the questionnaire scales had a normal distribution, we also performed comparisons using the Mann-Whitney $U$ test. The results for the two approaches were similar. Potentially important explanatory variables for physical health and mental health were included in a multivariate backward stepwise regression model. The variables for physical health were ESRD, diabetes, age, angina pectoris, amputation, impaired vision, smoking and education, and for

Table 1 Characteristics of diabetic and non-diabetic patients treated with dialysis and diabetic patients with normal kidney function

\begin{tabular}{|c|c|c|c|}
\hline & $\begin{array}{l}\text { Diabetic dialysis patients, } \\
n=38\end{array}$ & $\begin{array}{l}\text { Non-diabetic dialysis } \\
\text { patients, } n=40\end{array}$ & $\begin{array}{l}\text { Diabetic patients with normal kidney } \\
\text { function } n=38\end{array}$ \\
\hline \multicolumn{4}{|l|}{ Socio-demographics } \\
\hline Age & $63.6 \pm 13$ & $61.4 \pm 12$ & $60.2 \pm 13$ \\
\hline Sex $(\%$ male $)$ & 68 & 80 & 55 \\
\hline Education (short, medium, long) (\%) & $43: 41: 16$ & $28: 59: 13$ & $14: 46: 40 * *$ \\
\hline Working (part time or full time) (\%) & 5 & $24 *$ & $44 * * *$ \\
\hline Living with a partner or family (\%) & 54 & 60 & 46 \\
\hline \multicolumn{4}{|l|}{ Diabetes } \\
\hline Duration of diabetes (years) & $15.0(1-47)$ & - & $16.4(10-43)$ \\
\hline Type 1 diabetes & 29 & - & 34 \\
\hline $\mathrm{HbA}_{1 \mathrm{c}}(\%)$ & $7.1 \pm 1.5$ & $5.3 \pm 0.6^{* * *}$ & $8.0 \pm 1.0 * *$ \\
\hline Current foot lesions & 30 & $0 * * *$ & $13 * *$ \\
\hline Amputation & 18 & $0 * *$ & $5^{*}$ \\
\hline $\begin{array}{l}\text { Retinopathy (none, background, } \\
\text { proliferative) }\end{array}$ & $27: 20: 53$ & - & $37: 60: 3 * * *$ \\
\hline Maculopathy & 33 & - & $6 * * *$ \\
\hline Visual acuity $<0.3$ & 37 & - & $0 * * *$ \\
\hline \multicolumn{4}{|l|}{ Cardiovascular diseases and risk factors } \\
\hline History of myocardial infarction & 32 & 18 & 28 \\
\hline Angina pectoris & 18 & 10 & 12 \\
\hline BP (systolic) (mmHg) & $143 \pm 20$ & $135 \pm 19^{*}$ & $135 \pm 17^{*}$ \\
\hline BP (diastolic) (mmHg) & $74 \pm 10$ & $78 \pm 10$ & $80 \pm 9 *$ \\
\hline Cholesterol $(\mathrm{mmol} / \mathrm{l})$ & $4.5 \pm 1.2$ & $4.8 \pm 1.0$ & $4.3 \pm 0.9$ \\
\hline BMI & $25.2 \pm 4.0$ & $24.0 \pm 3.3$ & $29.6 \pm 6^{* * *}$ \\
\hline Currently smoking & 32 & 50 & 22 \\
\hline \multicolumn{4}{|l|}{ Dialysis } \\
\hline Duration of ESRD (years) & $1.90(0.30-17.7)$ & $2.12(0.15-14.9)$ & - \\
\hline Haemoglobin $(\mathrm{mmol} / \mathrm{l})$ & $7.6 \pm 0.8$ & $7.5 \pm 0.8$ & - \\
\hline Dialysis modality (haemodialysis \%) & 86 & 83 & - \\
\hline $\begin{array}{l}\mathrm{Kt} / \mathrm{V} \text { (haemodialysis patients) } \\
\text { (median, range) }\end{array}$ & $1.6(1.1-2.2)$ & $1.5(1.1-2.0)$ & - \\
\hline $\mathrm{Kt} / \mathrm{V}$ (peritoneal dialysis patients) & $2.3(2.1-3.2)$ & $2.7(1.8-3.7)$ & \\
\hline Haemodialysis access (fistula \%) & 50 & 50 & - \\
\hline
\end{tabular}

Values are presented as means $\pm \mathrm{SD}$, medians (range) or \%. School education: short, 7 years in municipal primary school; medium, 9 or 10 years in municipal primary school; long, grammar school

${ }^{*} p<0.05, * * p<0.01, * * * p<0.001$ vs diabetic dialysis patients 
mental health the selected variables were ESRD, diabetes, age, impaired vision, education, living with a partner or family, and working. For insignificant variables the estimate before exclusion from the model was reported. The relationship between physical health and mental health and the four subscales of the Locus of Control questionnaire were also investigated using univariate regression analyses, correcting for age and the presence of kidney disease. The comparison with the general population data used a regression method to weight the general population to the distribution of age, sex and school education in the patient group. In the general population, we found an interaction between age and education in the prediction of several SF-36 outcome variables. For this reason we included such interaction terms in our weighting scheme, but the effect of this was negligible. All statistical analyses were performed using the SAS 9.1 package (SAS institute, Cary, NC, USA).

\section{Results}

Complications, clinical, social and demographic data

Among the diabetic dialysis patients every third had previously suffered an acute myocardial infarction, every fourth had previously experienced a stroke, and every fifth patient had current angina pectoris (Table 1). Retinopathy (any), maculopathy and impaired vision were significantly more frequent among diabetic dialysis patients than diabetic controls (Table 1). The level of education was significantly lower in diabetic dialysis patients than in diabetic controls, and very few diabetic dialysis patients were employed (Table 1). Variables regarding the quality of the dialysis treatments (haemoglobin, $\mathrm{Kt} / \mathrm{V}$ and percentage of haemodialysis patients with an arterio-venous fistula) were similar in diabetic and non-diabetic patients. $\mathrm{HbA}_{1 \mathrm{c}}$ and $\mathrm{BMI}$ were significantly lower in diabetic dialysis patients than in diabetic controls. Apart from slightly longer diabetes duration in dialysis patients who did not complete the questionnaires, there were no significant differences in any of the variables in Table 1 between those who did and those who did not fill in the questionnaires (data not shown).

\section{Quality of life}

There were no significant differences in the scores of the kidney-specific part of the KDQOL-SF questionnaire between diabetic and non-diabetic patients (Table 2) The SF-36 scores in diabetic dialysis patients were significantly lower than those in the general population for the two component scores (PCS and MCS) and for all subscales except bodily pain (Table 3). The diabetic dialysis patients scored significantly
Table 2 KDQOL score in 38 diabetic and 40 non-diabetic patients treated with dialysis

\begin{tabular}{llll}
\hline & \multicolumn{2}{l}{ Patients treated with dialysis } & $p$ value \\
\cline { 2 - 3 } & $\begin{array}{l}\text { Diabetic } \\
(n=38)\end{array}$ & $\begin{array}{l}\text { Non-diabetic } \\
(n=40)\end{array}$ & $\begin{array}{l}\text { (Mann- } \\
\text { Whitney } \\
U \text { test })\end{array}$ \\
\hline Symptoms & & & 0.54 \\
Effect of kidney disease & $67(16)$ & $67(17)$ & 0.88 \\
Burden of kidney & $39(27)$ & $41(28)$ & 0.75 \\
$\quad$ disease & $14(29)$ & $28(40)$ & 0.17 \\
Work status & $87(15)$ & $82(21)$ & 0.31 \\
Cognitive function & $80(16)$ & $74(18)$ & 0.16 \\
Quality of social & & & \\
$\quad$ interaction & $63(45)$ & $66(32)$ & 0.87 \\
Sexual functioning & $66(19)$ & $68(21)$ & 0.77 \\
Sleep & $81(26)$ & $67(33)$ & 0.06 \\
Social support & $81(29)$ & $87(16)$ & 0.98 \\
Staff encouragement & $86(20)$ & $77(24)$ & 0.05 \\
Patient satisfaction & $68(11)$ & $66(12)$ & 0.46 \\
KDCS (summary score) & 68 &
\end{tabular}

Values are presented as means $\pm \mathrm{SD}$

lower in terms of physical function and physical health compared with both non-diabetic dialysis controls and diabetic controls, and in terms of role physical, general health, vitality and role emotional compared with diabetic controls. Mental health scores were similar in the three patient groups but were significantly lower than in the general Danish population.

In the multivariate analysis ESRD, diabetes and short education predicted poor physical health, and smoking, amputation and the presence of angina pectoris also tended to have a negative influence on physical health (Table 4).

There were no interactions between diabetes and any of the variables in the model or between ESRD and any of the variables in the model. The whole model explained $29 \%$ of the variance in physical health. Mental health was not significantly predicted by any of the selected variables, though living with a partner or family tended to have a positive influence on mental health (data not shown).

Locus of control

Diabetic dialysis patients scored significantly higher than the diabetic controls in the subscale 'chance' (15 vs 11, $p<$ 0.01 ) of the MHLC-C, whereas the scores of the subscales 'internal', 'doctors' and 'powerful others' were similar in the two groups. Compared with patients who spent a short or medium time in education, patients who spent a long time in education had a significantly lower belief in the three external subscales: 'chance' ( 9.8 vs $14.1, p<0.02$ ), 'doctors' (12.1 vs 14.4, $p<0.05)$ and 'powerful others' (6.1 vs $8.9, p<0.01)$. There were no significant differences in 
Table 3 Quality of life estimated by SF-36 scores in 38 diabetic and 40 non-diabetic patients treated with dialysis, 38 diabetic patients with normal kidney function and the general population

\begin{tabular}{lllll}
\hline & $\begin{array}{l}\text { Diabetic patients treated } \\
\text { with dialysis, } n=38\end{array}$ & $\begin{array}{l}\text { Non-diabetic patients } \\
\text { treated with dialysis, } \\
n=40\end{array}$ & $\begin{array}{l}\text { Diabetic patients with } \\
\text { normal kidney function, } \\
n=38\end{array}$ & $\begin{array}{l}\text { General population, } n=2248 \\
\text { (weighted to match sex, age, } \\
\text { and education) }\end{array}$ \\
\hline Physical function & $36 \pm 27$ & $57 \pm 28^{\mathrm{b}}$ & $73 \pm 30^{\mathrm{d}}$ & $82^{\mathrm{d}}$ \\
Role physical & $26 \pm 36$ & $41 \pm 43$ & $64 \pm 43^{\mathrm{c}}$ & $72^{\mathrm{d}}$ \\
Pain & $68 \pm 25$ & $71 \pm 26$ & $68 \pm 32$ & 75 \\
General health & $40 \pm 15$ & $47 \pm 20$ & $56 \pm 23^{\mathrm{b}}$ & $70^{\mathrm{d}}$ \\
Vitality & $47 \pm 19$ & $50 \pm 27$ & $60 \pm 26^{\mathrm{a}}$ & $70^{\mathrm{d}}$ \\
Social function & $75 \pm 28$ & $78 \pm 27$ & $82 \pm 27$ & $90^{\mathrm{c}}$ \\
Role emotional & $46 \pm 43$ & $49 \pm 42$ & $70 \pm 40^{\mathrm{a}}$ & $81^{\mathrm{d}}$ \\
Mental health & $74 \pm 19$ & $77 \pm 18$ & $76 \pm 20$ & $84^{\mathrm{b}}$ \\
PCS & $32 \pm 8$ & $39 \pm 10^{\mathrm{b}}$ & $45 \pm 13^{\mathrm{c}}$ & $49^{\mathrm{d}}$ \\
MCS & $50 \pm 10$ & $48 \pm 11$ & $51 \pm 11$ & $55^{\mathrm{b}}$
\end{tabular}

Values are presented as means $\pm \mathrm{SD}$

${ }^{\mathrm{a}} p<0.05,{ }^{\mathrm{b}} p<0.01,{ }^{\mathrm{c}} p<0.001,{ }^{\mathrm{d}} p<0.0001$ vs diabetic dialysis patients; calculated using the Mann-Whitney $U$ test for comparisons with matched study groups, and calculated using multiple regression controlling for sex, age and education for comparisons with the general population.

the 'internal' score between the two groups (long education: 28 vs short or medium education: 29). In univariate regression analyses of the effect of the four subscales on mental health (corrected for dialysis and age) we found that greater internal belief was associated with significantly better mental health (an increase in the mental health score of $4,95 \%$ CI $0.2-7.8$ per increase of 10 in internal score, $p<0.05$ ). In addition, significantly worse mental health was associated with a greater belief in 'chance' (changes in mental health of $-6.8,95 \% \mathrm{CI}-10.3$ to -3.2 per increase of $10, p<0.001$ ) and 'powerful others' (changes in mental health of $-7.8,95 \% \mathrm{CI}-14$ to -1.6 per increase of $10, p<$ $0.05)$. The loci of control scores were not associated with physical health scores (data not shown).

Table 4 Multivariate analysis of the predictors of physical health in diabetic and non-diabetic dialysis patients and diabetic patients with normal kidney function

\begin{tabular}{lll}
\hline & $\begin{array}{l}\text { Estimated change in } \\
\text { physical health score } \\
(95 \% \mathrm{CI}), n=104\end{array}$ & $p$ value \\
\hline ESRD & $-11(-15$ to -6$)$ & $<0.001$ \\
Diabetic vs non-diabetic & $-7(-12$ to -2$)$ & $<0.01$ \\
Education (long vs short & $6(2$ to 11$)$ & $<0.01$ \\
$\quad$ or medium) & $-6(-15$ to 2$)$ & 0.15 \\
Amputation & $-3(-7$ to 2$)$ & 0.20 \\
Smoking vs non-smoking & $-4(-10$ to 2$)$ & 0.22 \\
Angina pectoris & $-0.6(-2.4$ to 1.1$)$ & 0.47 \\
Age (per 10 years) & $-0.5(-10$ to 8$)$ & 0.97 \\
Impaired vision & & \\
\hline
\end{tabular}

\section{Discussion}

In this study of diabetic patients treated with chronic dialysis at a single centre, we found a high prevalence of diabetic complications and cardiovascular diseases among diabetic dialysis patients. The kidney-specific quality of life of the diabetic dialysis patients was similar to that of nondiabetic controls, whereas self-rated physical health was significantly reduced compared with that of the two wellmatched control groups (non-diabetic dialysis patients and diabetic patients with normal kidney function) and the general population.

Mental health was similar in the three patient groups, and although the scores were significantly worse than general population scores, the differences were not large.

The presence of ESRD, diabetes and a low level of education were significant negative predictors of physical health. Diabetic patients on dialysis had a significantly greater belief in chance in relation to their diabetes, and significantly fewer dialysis patients had a long school education compared with diabetic controls.

Strengths and weaknesses of the study

We compared diabetic dialysis patients with two matched control groups and the general population, which allowed us to separately evaluate the impact of diabetes and kidney disease on the quality of life of diabetic dialysis patients. The response rate to the questionnaires is comparable to other studies in the field, and demographic and clinic variables were similar among responders and non-responders. Although the sample size was relatively small, significantly findings regarding physical health and locus of control were 
observed. Data from a large survey in the general Danish population were used as a reference, because Danish individuals are known to have a higher self-rated health compared with other countries in Europe and the USA. Furthermore, these data were matched regarding age, sex, place of living and level of education.

Diabetic complications and cardiovascular diseases

In a large study of type 2 diabetic patients undergoing haemodialysis (4D study) Wanner and colleagues found a history of myocardial infarction, CABG or PCI in $23 \%$ and a history of stroke in $18 \%$ [5]. These frequencies are slightly lower than those found in the present study. This is probably due to the fact that the 4D study excluded patients who had received dialysis for more than 2 years and patients with very low or very high levels of cholesterol. These excluded patients with a long duration of ESRD and extreme cholesterol values might have had an even higher prevalence of both myocardial infarction and stroke. The prevalence rates of foot ulcer and amputation were similar to what has been reported in other studies in the field [3741]. Studies of both type 1 and type 2 diabetic patients also found that the presence of any retinopathy was associated with a higher risk of the development of ESRD [42, 43]. Our study confirmed the high prevalence of diabetic and cardiovascular complications in diabetic dialysis patient. It also underlines the importance of multi-factorial diabetic treatment and care, not only when patients are in the early stages of diabetic nephropathy or in the pre-dialysis state, but also when they have ESRD.

Kidney disease, physical health, mental health and quality of life

With respect to variables related to quality of dialysis treatment (haemoglobin, $\mathrm{Kt} / \mathrm{V}$ and percentage with an arteriovenous fistula) and the KDQOL, we found similar results among diabetic and non-diabetic dialysis patients. These observations suggest that dialysis is as effective in diabetic as in non-diabetic patients. However, diabetic patients receiving dialysis demonstrated a reduction in physical health, with limitations in daily activities such as climbing stairs, walking, bathing and dressing and reduced role functioning at work or at home as a result of physical health problems. It has previously been shown that physical exercise results in an increased physical function in dialysis patients with low PCS scores [44] and in patients with higher baseline PCS scores $[45,46]$. Intensified focus on physical training for diabetic patients in dialysis therefore seems relevant.

The scores for mental health were significantly lower in the three patient groups than in the general population.
However, the differences were small and there were no differences between the diabetic dialysis patients and the two control groups.

To our knowledge this is the first study to report the results of the KDQOL questionnaire in diabetic and nondiabetic dialysis patients matched in terms of age, dialysis modality and duration of ESRD. Studies that have investigated both diabetic and non-diabetic patients found similar results for the 11 subscales and the KDCS scores, with a trend towards higher scores in our study [25-28]. A Spanish study [47] compared quality of life of diabetic dialysis patients and unmatched non-diabetic patients using the SF-36 questionnaire and found significantly lower scores in seven out of eight subscales, but did not correct for the fact that the Spanish diabetic patients were significantly older than the non-diabetic patients $(61.9 \pm$ 13.5 vs $56.6 \pm 17$ years). In the Spanish study PCS and MCS scores were significantly reduced in diabetic compared with non-diabetic patients (34 vs 40 and 39 vs 42 , respectively). In a previous study of haemodialysis patients at our clinic, Molsted et al. found comparable scores on the eight subscales and an average physical health score of $37 \pm$ 11 and a mental health score of $50 \pm 11$ [48], which are very similar to the scores in the present study.

The mental health scores in this study are in contrast to the results reported in some studies [27, 47], but in accordance with a study of quality of life in chronic kidney disease (CKD) patients with different GFRs [26]. They found decreasing physical health with decreasing GFR, but a similar mental health score in the five CKD groups and the dialysis group in which the mental health score was $50.0 \pm 9$. In our study, having a chronic disease such as diabetes or ESRD decreases self-rated mental health by approximately half an SD compared with the general population. However, mental health was not influenced by the severity of the disease.

The PCS and MCS are standardised to the American general population, with a general population mean $\pm \mathrm{SD}$ of $50 \pm 10$. The mean mental health score in the Danish general population is higher, with an average of $55 \pm 10$. The mental health scores in this study are high by US standards, but this has been a general finding of Danish quality of life studies [48] and may be due to a culture-specific use of language or a general higher level of wellbeing in Denmark. The results underscore the value of a national comparison group.

\section{Predictors of quality of life}

In the multivariate regression analysis we found that being on dialysis was the most pronounced predictor of decreased physical health, followed by diabetes. However, serious diabetic complications (amputation and impaired vision) or 
angina pectoris tended to predict lower physical health but were not significant, a finding that might be due to a lack of statistical power in this study. From large studies in the general population it is well known that education is a significant predictor of quality of life, which is in accordance with the finding in this study. Although school education is not a modifiable characteristic, the observed prediction of physical health, the differences in time spent in education between diabetic patients on dialysis and diabetic controls and the differences in locus of control are potentially useful for the identification of those diabetic patients who need special attention to improve treatment adherence and thereby reduced the risk of ESRD.

Regarding mental health, we found no significant effects of any of the selected clinical or socio-demographic variables, and the model only explained a small part of the total variation in mental health. However, the patients' beliefs regarding control of diabetes were significant predictors of mental health. We found a high internal control belief in the two diabetic patient groups and a significantly higher belief in chance among diabetic dialysis patients. The associations of high internal belief and low external belief (chance and powerful others) with better mental health score are in accordance with the expected pattern for locus of control in diabetes but since our study is cross-sectional, no causal inference can be made.

\section{Conclusion}

Diabetic dialysis patients are characterised by a high prevalence of diabetic complications, reduced self-rated physical health, but relatively good mental health. The finding of reduced physical function enables us to focus on the importance of physical training. The association between internal locus of control and good mental health suggest that interventions to strengthen internal locus of control might also be valuable.

Implementation of so-called 'diabetes schools' and 'kidney schools', which offer more intensive information and education for the patient regarding treatment and selfcare of the disease, have become more common and may show the way to improve patient adherence, quality of life, physical function and survival.

\footnotetext{
Acknowledgements We thank the Danish Diabetes Association and the Danish Kidney Association, who supported the work by funding awarded to V. R. Sørensen.
}

Duality of interest The authors declare that there is no duality of interest associated with this manuscript.

\section{References}

1. Sorensen VR, Hansen PM, Heaf J, Feldt-Rasmussen B (2006) Stabilized incidence of diabetic patients referred for renal replacement therapy in Denmark. Kidney Int 70:187-191

2. Feldt-Rasmussen B (2006) Is there a need to optimize glycemic control in hemodialyzed diabetic patients? Kidney Int 70:1392-1394

3. Kalantar-Zadeh K, Kopple JD, Regidor DL et al (2007) A1C and survival in maintenance hemodialysis patients. Diabetes Care 30:1049-1055

4. Sorensen VR, Mathiesen ER, Heaf J, Feldt-Rasmussen B (2007) Improved survival rate in patients with diabetes and end-stage renal disease in Denmark. Diabetologia 50(5):922-929

5. Wanner C, Krane V, Marz W et al (2004) Randomized controlled trial on the efficacy and safety of atorvastatin in patients with type 2 diabetes on hemodialysis (4D study): demographic and baseline characteristics. Kidney Blood Press Res 27:259-266

6. Rajagopalan S, Dellegrottaglie S, Furniss AL et al (2006) Peripheral arterial disease in patients with end-stage renal disease: observations from the Dialysis Outcomes and Practice Patterns Study (DOPPS). Circulation 114:1914-1922

7. Adler AI, Stratton IM, Neil HA et al (2000) Association of systolic blood pressure with macrovascular and microvascular complications of type 2 diabetes (UKPDS 36): prospective observational study. BMJ 321:412-419

8. Brenner BM, Cooper ME, de Zeeuw D et al (2001) Effects of losartan on renal and cardiovascular outcomes in patients with type 2 diabetes and nephropathy. N Engl J Med 345:861-869

9. Gaede P, Vedel P, Parving HH, Pedersen O (1999) Intensified multifactorial intervention in patients with type 2 diabetes mellitus and microalbuminuria: the Steno type 2 randomised study. Lancet 353:617-622

10. Gaede P, Vedel P, Larsen N, Jensen GV, Parving HH, Pedersen O (2003) Multifactorial intervention and cardiovascular disease in patients with type 2 diabetes. N Engl J Med 348:383-393

11. Parving $\mathrm{HH}$, Lehnert $\mathrm{H}$, Brochner-Mortensen J, Gomis R, Andersen S, Arner P (2001) The effect of irbesartan on the development of diabetic nephropathy in patients with type 2 diabetes. N Engl J Med 345:870-878

12. Rossing K, Jacobsen P, Pietraszek L, Parving HH (2003) Renoprotective effects of adding angiotensin II receptor blocker to maximal recommended doses of ACE inhibitor in diabetic nephropathy: a randomized double-blind crossover trial. Diabetes Care 26:2268-2274

13. Chapman RH, Benner JS, Petrilla AA et al (2005) Predictors of adherence with antihypertensive and lipid-lowering therapy. Arch Intern Med 165:1147-1152

14. Lerman I (2005) Adherence to treatment: the key for avoiding long-term complications of diabetes. Arch Med Res 36:300-306

15. Rubin RR (2005) Adherence to pharmacologic therapy in patients with type 2 diabetes mellitus. Am J Med 118:27S-34S

16. Fagerudd J, Forsblom C, Pettersson-Fernholm K, Groop PH (2004) Implementation of guidelines for the prevention of diabetic nephropathy. Diabetes Care 27:803-804

17. Chao J, Nau DP, Aikens JE, Taylor SD (2005) The mediating role of health beliefs in the relationship between depressive symptoms and medication adherence in persons with diabetes. Res Soc Adm Pharm 1:508-525

18. Schachinger H, Hegar K, Hermanns N et al (2005) Randomized controlled clinical trial of Blood Glucose Awareness Training (BGAT III) in Switzerland and Germany. J Behav Med 28:587-594

19. Reynaert C, Janne P, Donckier J et al (1995) Locus of control and metabolic control. Diabete Metab 21:180-187

20. O'Hea EL, Grothe KB, Bodenlos JS, Boudreaux ED, White MA, Brantley PJ (2005) Predicting medical regimen adherence: the 
interactions of health locus of control beliefs. J Health Psychol 10:705-717

21. Tillotson LM, Smith MS (1996) Locus of control, social support, and adherence to the diabetes regimen. Diabetes Educ 22:133-139

22. Schlenk EA, Hart LK (1984) Relationship between health locus of control, health value, and social support and compliance of persons with diabetes mellitus. Diabetes Care 7:566-574

23. Wallston KA (2005) The validity of the multidimensional health locus of control scales. J Health Psychol 10:623-631

24. Danish National Institute of Public Health (2006) The national health and morbidity survey 2005. Danish National Institute of Public Health, Copenhagen

25. Hays RD, Kallich JD, Mapes DL, Coons SJ, Carter WB (1994) Development of the Kidney Disease Quality of Life (KDQOL) instrument. Qual Life Res 3:329-338

26. Gorodetskaya I, Zenios S, McCulloch CE et al (2005) Healthrelated quality of life and estimates of utility in chronic kidney disease. Kidney Int 68:2801-2808

27. Fukuhara S, Lopes AA, Bragg-Gresham JL et al (2003) Health-related quality of life among dialysis patients on three continents: the Dialysis Outcomes and Practice Patterns Study. Kidney Int 64:1903-1910

28. Mapes DL, Lopes AA, Satayathum S et al (2003) Health-related quality of life as a predictor of mortality and hospitalization: the Dialysis Outcomes and Practice Patterns Study (DOPPS). Kidney Int 64:339-349

29. Valdes C, Garcia-Mendoza M, Rebollo P, Ortega T, Ortega F (2006) Mental health at the third month of haemodialysis as a predictor of short-term survival. Nephrol Dial Transplant 21:3223-3230

30. Bjorner JB, Damsgaard MT, Watt T, Groenvold M (1998) Tests of data quality, scaling assumptions, and reliability of the Danish SF36. J Clin Epidemiol 51:1001-1011

31. Bjorner JB, Thunedborg K, Kristensen TS, Modvig J, Bech P (1998) The Danish SF-36 Health Survey: translation and preliminary validity studies. J Clin Epidemiol 51:991-999

32. Bro S, Bjorner JB, Tofte-Jensen P et al (1999) A prospective, randomized multicenter study comparing APD and CAPD treatment. Perit Dial Int 19:526-533

33. Molsted S, Heaf J, Prescott L, Eidemak I (2005) Reliability testing of the Danish version of the Kidney Disease Quality of Life Short Form. Scand J Urol Nephrol 39:498-502

34. Ware JE, Kosinski M (2001) Interpreting SF-36 summary health measures: a response. Qual Life Res 10:405-413

35. Bjorner JB, Damsgaard MT, Watt T et al (1997) Danish manual for the SF-36. Danish National Institute of Health, Copenhagen (in Danish)
36. Christensen AJ, Turner CW, Smith TW, Holman JM, Jr, Gregory MC (1991) Health locus of control and depression in end-stage renal disease. J Consult Clin Psychol 59:419-424

37. Game FL, Chipchase SY, Hubbard R, Burden RP, Jeffcoate WJ (2006) Temporal association between the incidence of foot ulceration and the start of dialysis in diabetes mellitus. Nephrol Dial Transplant 21:3207-3210

38. Koch M, Trapp R, Kulas W, Grabensee B (2004) Critical limb ischaemia as a main cause of death in patients with end-stage renal disease: a single-centre study. Nephrol Dial Transplant 19:25472552

39. Yasuhara H, Naka S, Yanagie H, Nagawa H (2002) Influence of diabetes on persistent nonhealing ischemic foot ulcer in end-stage renal disease. World J Surg 26:1360-1364

40. Locking-Cusolito H, Harwood L, Wilson B et al (2005) Prevalence of risk factors predisposing to foot problems in patients on hemodialysis. Nephrol Nurs J 32:373-384

41. Rith-Najarian S, Gohdes D (2000) Preventing amputations among patients with diabetes on dialysis. Diabetes Care 23:1445-1446

42. Cusick M, Chew EY, Hoogwerf B et al (2004) Risk factors for renal replacement therapy in the Early Treatment Diabetic Retinopathy Study (ETDRS), Early Treatment Diabetic Retinopathy Study Report No. 26. Kidney Int 66:1173-1179

43. Parving HH, Mogensen CE, Thomas MC, Brenner BM, Cooper ME (2005) Poor prognosis in proteinuric type 2 diabetic patients with retinopathy: insights from the RENAAL study. QJM 98:119126

44. Painter P, Carlson L, Carey S, Paul SM, Myll J (2000) Lowfunctioning hemodialysis patients improve with exercise training. Am J Kidney Dis 36:600-608

45. Molsted S, Eidemak I, Sorensen HT, Kristensen JH (2004) Five months of physical exercise in hemodialysis patients: effects on aerobic capacity, physical function and self-rated health. Nephron Clin Pract 96:c76-c81

46. Painter P, Carlson L, Carey S, Paul SM, Myll J (2000) Physical functioning and health-related quality-of-life changes with exercise training in hemodialysis patients. Am J Kidney Dis 35:482492

47. Martinez-Castelao A, Gorriz JL, Garcia-Lopez F, Lopez-Revuelta K, De AF, Cruzado JM (2004) Perceived health-related quality of life and comorbidity in diabetic patients starting dialysis (CALVIDIA study). J Nephrol 17:544-551

48. Christensen K, Herskind AM, Vaupel JW (2006) Why Danes are smug: comparative study of life satisfaction in the European Union. BMJ 333:1289-1291 